\title{
Typhonium flagelliforme induces apoptosis in CEMss cells via activation of caspase-9, PARP cleavage and cytochrome c release: Its activation coupled with G0/G1 phase cell cycle arrest.
}

\begin{abstract}
Ethnopharmacological relevance: The plant Typhonium flagelliforme (TF), commonly known as 'rodent tuber' in Malaysia, is often used as traditional remedy for cancer, including leukemia. Aim of the study:Wehad previously identified morphologically that the linoleic acid rich fraction (DCM/F7) from the tubers of this plant induces selective anti-proliferative effects and apoptosis in CEMss cells. In this present study, we subjected the same DCM/F7 fraction to cell based activity analyses in order to determine the possible mechanism of cell death in leukemic CEMss cells in vitro. Materials and methods: Extraction of Typhonium flagelliforme tuber has done and fractionation has been done by vacuum liquid column chromatography. The anti-proliferative activity was assayed using MTT and the apoptosis detection was done by Annexin V and DNA laddering assay. Colorimetric caspase assay and immunoblot analysis were employed to detect the expression of protein associated with cell death. Cell cycle analysis was done using flow cytometry. Results: We found that the cancer inhibitory effect of the DCM/F7 fraction in CEMss cells was $3 \pm 0.08 \mathrm{~g} / \mathrm{ml}$ (IC50). An early apoptotic induction in CEMss cells was observed by Annexin V assay, which showed a clear dose-dependent DNA fragmentation being observed in gel electrophoresis at 10 and $20 \mathrm{~g} / \mathrm{ml}$. The DCM/F7 fraction at 3g/ml significantly arrested CEMss cells at G0/G1 phase ( $<<0.05$ ). A constant but increasing pattern-related Sub-G0/G1 index was observed between 12 and 72 $\mathrm{h}$ treatment. In relation to this, we further investigated the biochemical events leading to cell death and found that the DCM/F7 fraction increased the cellular levels of caspase-3 and -9 on treated cells. Our results indicated that cytochrome $\mathrm{c}$ from mitochondria into the cytosol increased gradually as the DCM/F7 concentration increases, which later lead to the subsequent cleavage of PARP in to $85 \mathrm{kDa}$ fragments. On the contrary, Bcl-2 protein was found to decrease concomitantly during treatment. Conclusions: Collectively, results presented in this study demonstrated that the DCM/F7 fraction inhibited the proliferation of leukemia cells, leading to the programmed cell death, which was confirmed to be through the mitochondrial pathway.
\end{abstract}

Keyword: Typhonium flagelliforme; Leukemia; Cell cycle; Pathway Apoptosis. 